OPEN ACCESS

Edited by:

Gokhan Zengin,

Selcuk University, Turkey

Reviewed by:

Sengul Uysal,

Erciyes University, Turkey

Zhao Aihua,

Shanghai Jiao Tong University, China

*Correspondence:

Shan Cao

37493592@qq.com

Lixiao Liu

18918753950@163.com

${ }^{+}$These authors have contributed equally to this work

Specialty section:

This article was submitted to Ethnopharmacology,

a section of the journal

Frontiers in Pharmacology

Received: 06 December 2021

Accepted: 10 January 2022

Published: 08 February 2022

Citation:

Wang $D$, Zhao $L$, Hao $Z$, Huang $Y$, Liao Y, Wang L, Zhang J, Cao $S$ and Liu L (2022) High-Throughput and

Untargeted Metabolic Profiling

Revealed the Potential Effect and Mechanisms of Paeoniflorin in Young

Asthmatic Rats.

Front. Pharmacol. 13:829780.

doi: 10.3389/fphar.2022.829780

\section{High-Throughput and Untargeted Metabolic Profiling Revealed the Potential Effect and Mechanisms of Paeoniflorin in Young Asthmatic Rats}

\author{
Dan Wang ${ }^{1+}$, Li Zhao ${ }^{2+}$, Zhiyan Hao ${ }^{1}$, Ying Huang ${ }^{1}$, Yang Liao ${ }^{1}$, Lingli Wang ${ }^{1}$, Jinfeng Zhang ${ }^{1}$, \\ Shan $\mathrm{Cao}^{1 *}$ and Lixiao Liu $^{1 *}$ \\ ${ }^{1}$ Department of Pediatrics, Shanghai Pudong Hospital, Fudan University Pudong Medical Center, Shanghai, China, ${ }^{2}$ Sanya \\ Women and Children's Hospital Managed by Shanghai Children's Medical Center, Sanya, China
}

Paeoniflorin (PF) is a multi-target monoterpenoid glycoside and possesses broad pharmacological functions, e.g., anti-inflammation, anti-depression, antitumor, abirritation, neuroprotection, antioxidant, and enhancing cognitive and learning ability. PF has gained a large amount of attention for its effect on asthma disease as the growth rate of asthma has increased in recent years. However, its mechanism of action on asthma is still unclear. In this study, we have explored the action mechanism of PF on asthma disease. Furthermore, high-throughput untargeted metabolic profiling was performed through ultraperformance liquid chromatography/electrospray ionization quadruple time-of-flight high-definition mass spectrometry (QA) UPLC-Q/TOF-MS combined with pattern recognition approaches and pathway analysis. A total of 20 potential biomarkers were discovered by UPLC/MS and urine metabolic profiling. The key pathways including the citrate cycle (the TCA cycle), pyrimidine metabolism, pentose phosphate pathway, tyrosine metabolism, and tryptophan metabolism were affected by PF. In conclusion, we have discovered metabolite biomarkers and revealed the therapeutic mechanism of PF based on liquid chromatography coupled with mass spectrometry untargeted metabolomics. The untargeted metabolomics combined with UPLC-MS is a useful tool for exploring the therapeutic mechanism and targets of PF in the treatment of asthma. Metabolomics combined with UPLC-MS is an integrated method to explore the metabolic mechanism of $\mathrm{PF}$ in the treatment of asthma rats and to reveal the potential targets, providing theoretical support for the study of the treatment of PF.

Keywords: metabolomics, metabolites, pathways, biomarker, action mechanism 3

\section{INTRODUCTION}

Asthma is characterized by chronic airway inflammation, airway hyperresponsiveness, and airway remodeling and is a serious public health problem in the world (Chiu et al., 2021). At present, the inhaled corticosteroids are the standard treatment for persistent asthma, but its antioxidant effect is not ideal (Koh et al., 2021; Werder et al., 2021). This treatment strategy has serious adverse reactions, such as headache, tremor, palpitations, and heart failure (Bush et al., 2021; Jin et al., 2021). In recent years, herbal medicine has received more and more attention due to its wide range of pharmacological effects, low toxicity, and few adverse reactions. Many herbal medicines and their active ingredients can effectively 
relieve asthma attacks and have become a hot spot for asthma treatment. Paeoniflorin (PF) is the major bioactive ingredient derived from Paeonia lactiflora Pall., Paeonia suffruticosa Andr., or Paeonia veitchii Lynch, which have been widely used for cardiovascular disease, cerebrovascular disease, and liver disease. $\mathrm{PF}$ is the main active ingredient of the commonly used Paeonia lactiflora. In recent years, it has been found that PF has anti-inflammatory, antitumor, antibacterial, antiviral, immune regulation, and scavenging free radicals and other pharmacological effects and has less toxic and side effects.

Paeoniflorin, a monoterpenoid glycoside, is derived from Paeonia lactiflora Pall., Paeonia suffruticosa Andr., or Paeonia veitchii Lynch which has been used in traditional medical applications for more than 2000 years. Recent works have showed that it has a wide range of pharmacologic activities, including anti-depression, anti-inflammatory, anti-oxidation, anti-apoptosis, antitumor, and maintaining mitochondrial function (Chen et al., 2021; Han et al., 2021). Interestingly, previous scientific evidences suggested that it possesses promising anticancer activities on diverse tumors/cancers (Yu et al., 2021; Zhao et al., 2021). Recently, growing attention has been paid to explore to relieve asthma attack function of PF (Jiao et al., 2021; Wu et al., 2021). It has been demonstrated to have potent anti-asthma activity in various types of mouse or rat models (Shou et al., 2019). Even though the concrete mechanisms are still not fully clarified, it is speculated to be associated with increasing the levels of monoaminergic neurotransmitters, inhibiting the overactivation of the HPA axis, promoting the neurogenesis and neuroplasticity, suppressing the neuroinflammation reaction, enhancing neuroprotection, etc (Fan et al., 2020; Wei et al., 2020; Liu et al., 2021). The numerous studies have focused on the in-depth mechanism and attempted to investigate the efficacy of PF in asthma treatment (Guo et al., 2021; Ma et al., 2021).

Metabolomics as an analytical strategy has been used to reveal the relationship between the chemical component and endogenous metabolic biomarkers (Sun et al., 2019; Fang et al., 2020; Qiu et al., 2020). In short, through monitoring metabolic trajectories and changes in metabolites caused by external factors via metabolomics (Liang et al., 2015a; Liang et al., 2015b), the natural product is used to characterize and identify in vivo metabolites to reveal the effect and mechanisms (Liang et al., 2016a; Liang et al., 2016b; Zhang et al., 2019a; Zhang et al., 2019b; Xie et al., 2019; Zhang et al., 2020). Furthermore, the potential molecular mechanisms corresponding to the anti-asthma effects of paeoniflorin are lacking which should be focused on further studies. In this study, ovalbumin (OVA) was used to prepare a mouse asthma model and explore the effect and mechanism of PF on an asthma rat. Our present work on anti-asthma effects of PF would be beneficial for the further molecular mechanism study of PF in the future.

\section{METHODS AND MATERIALS}

\section{Chemicals and Reagents}

Paeoniflorin was supplied by Chengdu PUSH Bio. Technology Co., Ltd. (batch number PS186203-01), and the HPLC chromatography is shown in Supplementary Material, Figure S1. Wahaha pure water was supplied by Hangzhou Wahaha Group Co., Ltd. Estradiol benzoate injection was supplied by Animal Pharmaceutical Hangzhou, China (batch number 11212511). Watson's distilled water was supplied by Watson's Food \& Beverage Co., Ltd. (Guangzhou, China). Acetonitrile (HPLC grade) and methanol (HPLC grade) were supplied by Fisher (United States). Leucine enkephalin was supplied by Sigma-Aldrich (MO, United States). Other chemicals and reagents were of analytical purity.

\section{Animals}

Wistar rats, 4 weeks old, weighing $85-100 \mathrm{~g}$, were obtained from the Animal Center of Fudan University Pudong Medical Center. The room temperature and relative humidity were controlled at the range of $22-26^{\circ} \mathrm{C}$ and $35-45 \%$, respectively, with a $12 \mathrm{~h}$ light/ dark cycle. Prior to the experiment, all rats were put into the metabolism cages and allowed them to adapt to the environment for 7 days. Rats were given free access to water and normal food during this period. Then, we divided the rats into three groups stochastically: the control group (Con), the asthma model group (Mod), and the PF group (GY). The processes of the experiment were ratified by the Animal Care and Ethics Committee at the Fudan University Pudong Medical Center. In the light of the declaration of Helsinki, all experiments were carried out.

\section{Preparation of Juvenile Asthmatic Rats}

The young rats were divided into three groups according to the random number method: the control group, the model group, and the PF group, each with 10 rats. According to the method in the literature (Han et al., 2017), a juvenile asthma rat model was prepared. Except the control group, the other groups were intraperitoneally injected with $0.2 \mathrm{ml}$ OVA suspension (OVA $20 \mu \mathrm{g}+2 \mathrm{mg}$ hydrogen) on days 1,8 , and 15 . Alumina gel was sensitized, and the control group was injected with the same amount of normal saline. From the 16th day, the pups of the model group and treatment group were given a $4 \%$ OVA normal saline nebulized inhalation challenge once a day for 7 days. The pups in the control group were given the same amount of saline inhalation. After 1 hour, the pups of the control group and model group were intraperitoneally injected with normal saline, and the administration group was intraperitoneally injected with $9 \mathrm{mg} / \mathrm{kg}$ of $\mathrm{PF}$.

\section{Urine Sample Preparation}

The urine samples were collected and then centrifuged at 13,000 rpm/ $\min$ in $4^{\circ} \mathrm{C}$ for $10 \mathrm{~min}$. Before the UPLC-MS analysis, the supernatants were stored at $-80^{\circ} \mathrm{C}$. Before analysis, the urine samples were thawed in an ice bath; For analysis, $500 \mu \mathrm{L}$ urine and $500 \mu \mathrm{L}$ ultra-pure water were taken, vortexed for $30 \mathrm{~s}$, centrifuged at $13,000 \mathrm{rpm}$ for $15 \mathrm{~min}$ at $4^{\circ} \mathrm{C}$, and filtered over $0.22 \mu \mathrm{m}$; the supernatant was injected into the UPLC-MS.

\section{Chromatography}

The separation was accomplished through the ACQUITY UPLCTM phenomenex column $(2.1 \mathrm{~nm} \times 50 \mathrm{~mm}, 1.7 \mu \mathrm{m})$, controlling the column temperature at $40^{\circ} \mathrm{C}$. The optimal mobile phase contained (A) acetonitrile with $0.1 \%$ formic acid and (B) water with $0.1 \%$ formic acid. The detailed chromatographic conditions are in Supplementary Table S1. 


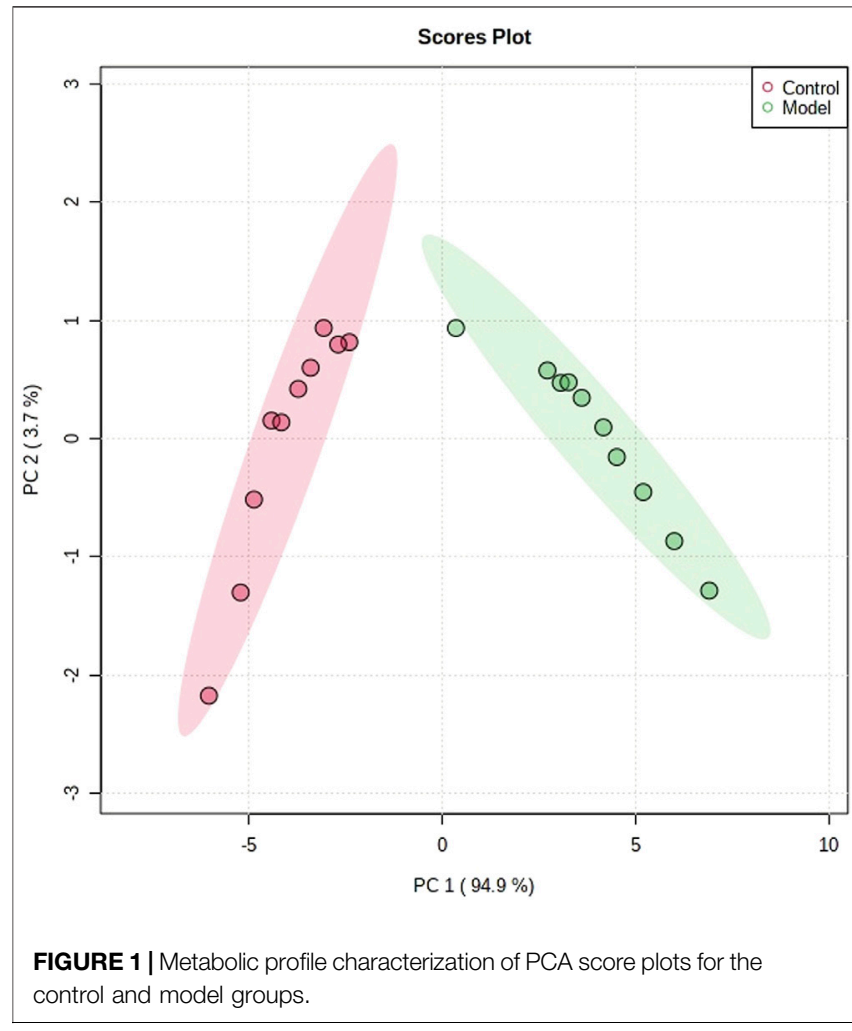

\section{Mass Spectrometry Analysis}

High-resolution mass spectrometry was performed on a TOF MS/MS system (Waters Corporation, United States) equipped with an electrospray ionization (ESI) source in both positive- and negative-ion modes. The optimized conditions were as follows: positive mode parameters: ion source temperature $110^{\circ} \mathrm{C}$, capillary voltage $3000 \mathrm{~V}$, cone voltage $30 \mathrm{~V}$, extraction cone voltage $5.0 \mathrm{~V}$, desolvation temperature $350^{\circ} \mathrm{C}$, cone gas flow $50 \mathrm{~L} / \mathrm{h}$, and desolvation gas flow $800 \mathrm{~L} / \mathrm{h}$; negative mode parameters: capillary voltage $2800 \mathrm{~V}$, cone voltage $40 \mathrm{~V}$, and all other parameters the same as in the positive-ion mode.

\section{Biomarker Identification}

The metabolic profiles (.raw data) obtained by using the UPLCMS system were imported into Progenesis QI software (V2.1, Waters Corporation, MA) for alignment, peak picking, and normalization. Then, resultant data matrices (.usp data) were opened by Ezinfo software (V3.0) for multivariate statistical analysis through pattern recognition methods. Metabolic data matrices between the control group and PF group were analyzed by OPLS-DA. A preliminary VIP value was selected for primary screening of potential biomarkers. The secondary screening was carried out according to the principle that each metabolite in the control and model groups had a significant difference $(p<0.05)$ which could be considered to be the possible biomarkers. Then, the mol file and secondary mass spectrometry information were matched. Finally, the urine biomarkers of asthma were confirmed.

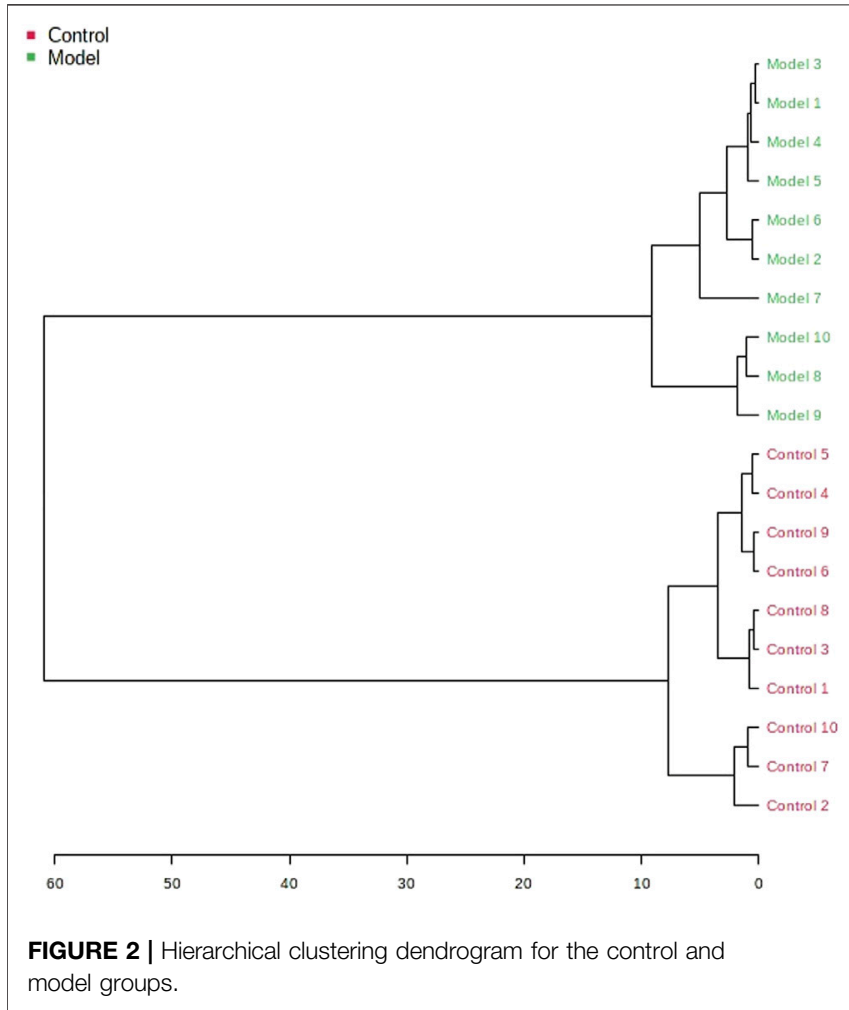

\section{Metabolic Pathway Analysis}

The KEGG, HMDB, or the name of the compound of the detected biomarker was introduced to the website http://www. metabioanalyst.ca for metabolic pathway analyses. The metabolomic pathway related to metabolites was found by analyzing the topological characteristics of the pathway. The metabolic pathways related to the ECB model are obtained and mapped, and then, the schematic diagram of each metabolic pathway was obtained. The impact threshold was set to 0.10 . Any pathway beyond this threshold was classified as a potential target pathway.

\section{Statistical Analysis}

Data was conveyed by Student's t-test and expressed as means \pm $\mathrm{SD}$. Differences in the average value were calculated statistically significant, and $p<0.05$ was considered statistically significant and $p<0.01$ meaning an extremely significant difference.

\section{RESULTS}

\section{Metabolic Profiling Analysis}

The obtained UPLC-MS/MS urine metabolic spectrum data were entered into Progenesis QI for chromatographic peak alignment, data normalization, peak extraction, and multivariate statistical analysis. PCA of the data was performed by the MetaboAnalyst software module, and it was determined whether the model creation resulted in changes in endogenous components. The PCA pattern recognition was performed on the control group's urine metabolic profile and the model group (Figure 1). PCA was conducted using urine sample data, and the final model revealed that the data profile 


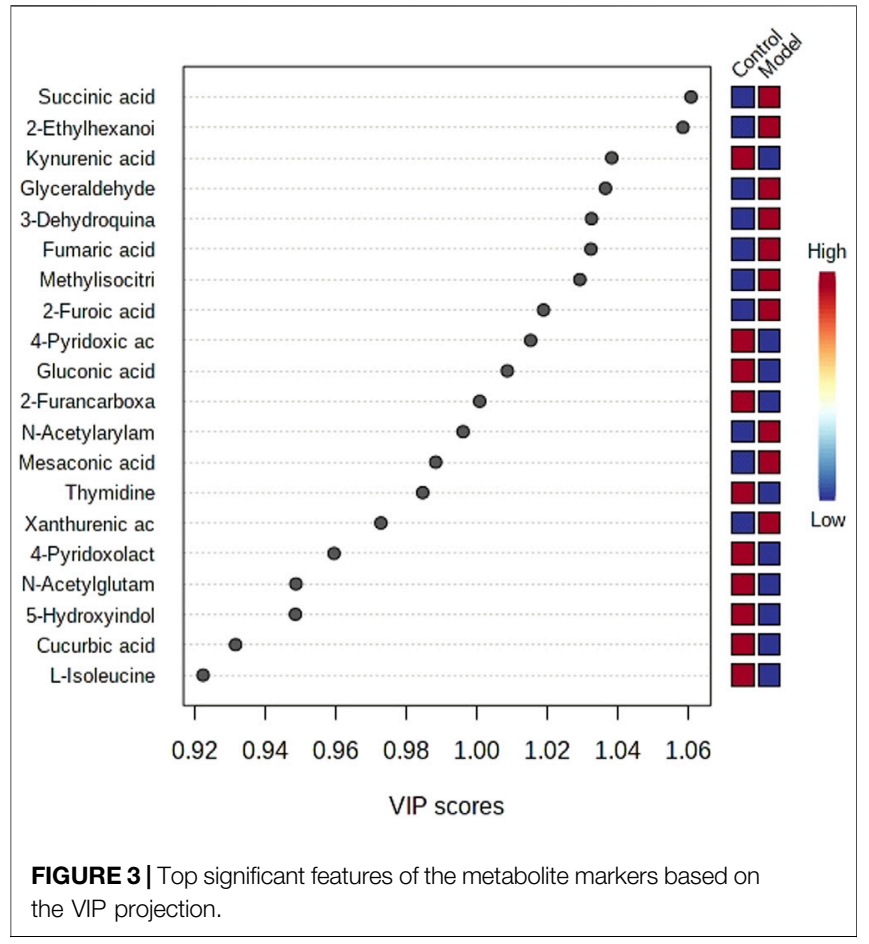

of the model group was far away from that of the control group, which indicated a significant change in the metabolic network in the model group (Figure 1). To further distinguish the differences between different groups, hierarchical clustering dendrogram analysis was performed on urine metabolites in the two groups (Figure 2). There are clear differences between the control and model groups, indicating that the model group's metabolism has changed after asthma. The VIP-plot is employed to screen potential biomarkers (Figure 3), the student's t-test is applied to analyze the difference ions with VIP $>1$, and the normalized abundance is statistically significant $(p<0.05)$; these important difference ions are considered as potential biomarkers, combining MS and MS/MS structural information, including gluconic acid, 2-furoic acid, 2ethylhexanoic acid, glyceraldehyde, cucurbic acid, $\mathrm{N}$-acetylarylamine, and fumaric acid, and finally, these potential biomarkers were found to be closely related to asthma (Supplementary Table S2).

\section{Identification of Urine Biomarkers}

A UPLC-MS/MS high-throughput analyzer coupled with Progenesis QI was used to determine the precise molecular mass and to generate MS/MS data for the structural identification of biomarkers. MetaboAnalyst was used to analyze the blood metabolic profiles of rats in the model and control groups, and hierarchical clustering dendrogram diagrams, which directly reflect the contribution of each component to the change in the metabolic profile, were created (Figure 4) to highlight the maximum difference between groups. The selected components were identified by determining their relative molecular weight by primary MS and obtaining their structural fragment information. A total of 20 potential biomarkers were collected using multiple databases, including the HMDB, KEGG, and METLIN, and the expression levels of the potential biomarker in the control and model groups are showed in Figure 4.

\section{Metabolic Pathway Analysis}

MetPA was used to construct and analyze metabolic pathways; the species was set to rat, and the HMDB numbers of the potential metabolites were entered for this pathway analysis. Using topological analysis, the cutoff value of the metabolic pathway influence was set to 0.01 , and pathways with a value greater than 0.01 were selected as potential key metabolic pathways. A total of 5 metabolic pathways were identified as related to asthma, including the citrate cycle (TCA cycle), pyrimidine metabolism, pentose phosphate pathway, tyrosine

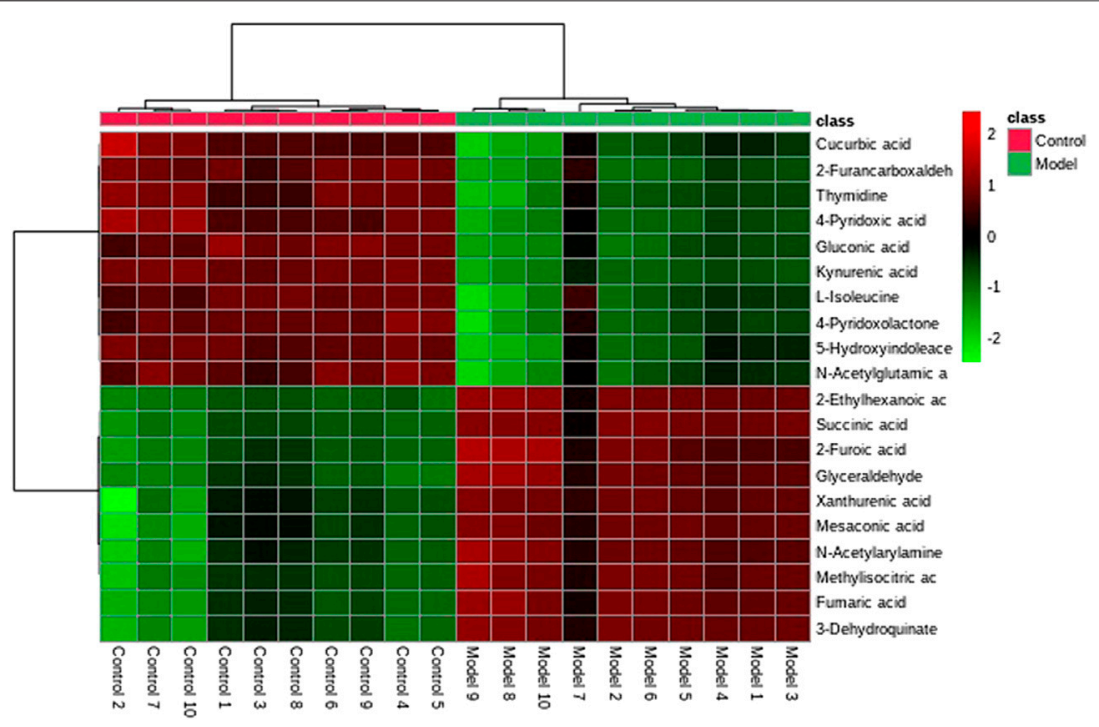

FIGURE 4 | Hierarchical clustering of the differential metabolites in the control and model groups. 

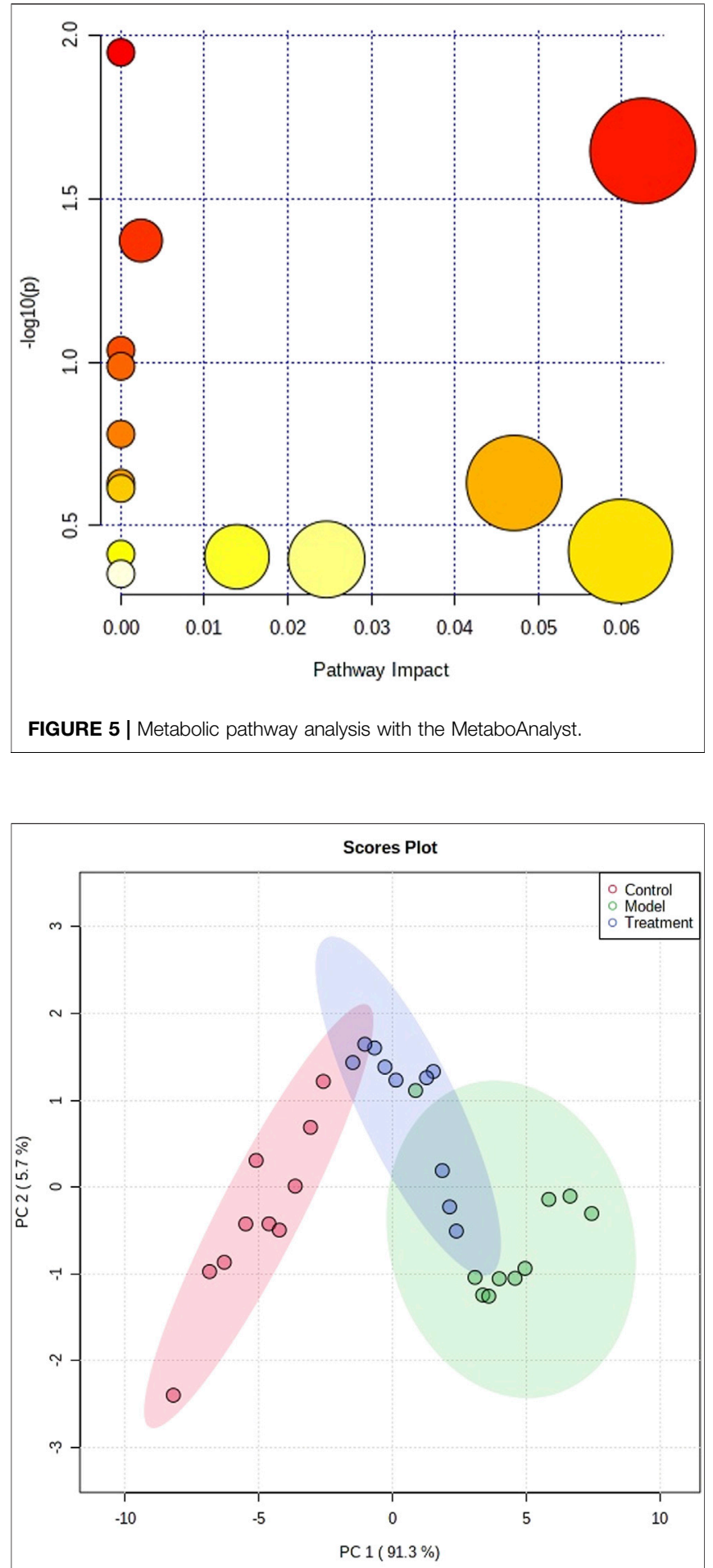

FIGURE 6 | PCA score plots of multivariate data analysis.

metabolism, and tryptophan metabolism (Figure 5; Supplementary Table S3). Therefore, the above metabolic pathways were identified as target pathways. The results showed that these biomarkers are involved in multiple metabolic pathways which were closely related to the asthma model.

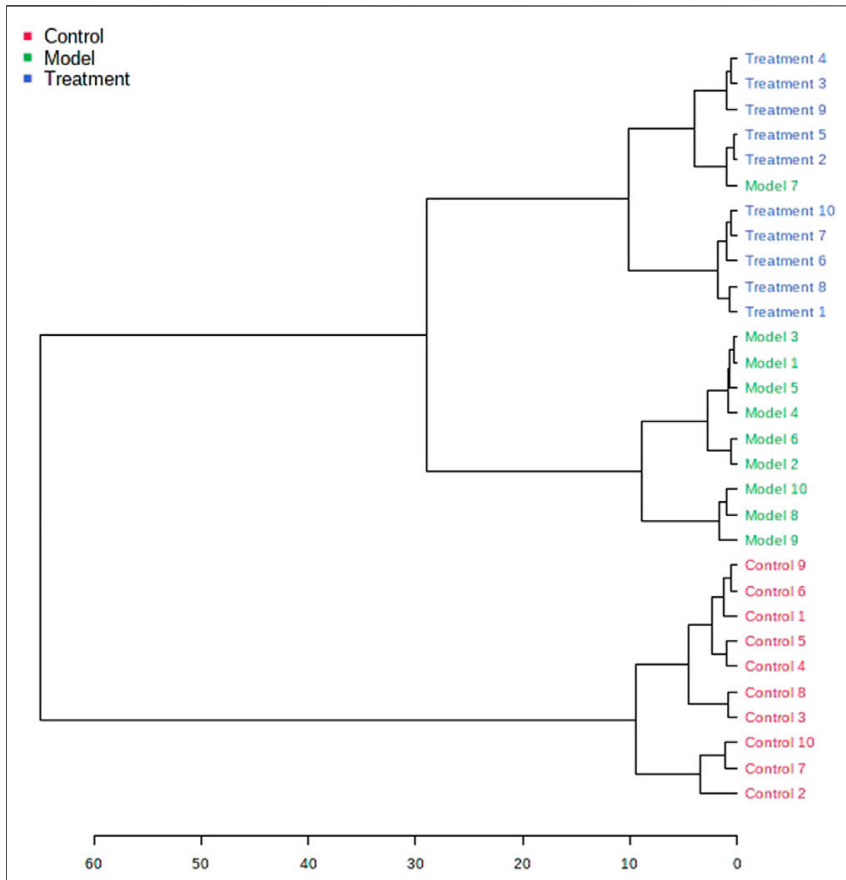

FIGURE 7 | Hierarchical clustering dendrogram for the control, model, and treatment groups.

\section{Therapeutic Effect of PF on Asthma Model Rats}

The metabolic profile of urine at the last day of administration was input into the Progenesis QI software for processing. Subsequently, these ions were processed by MetaboAnalyst to obtain score plots (Figure 6) that can reflect the trend among groups. The control group and the asthma model group showed obvious clustering and separation. After the administration, the model rats had a tendency approach to the control group. By analyzing the trend of the urine biomarkers, we found that PF can affect the microbiological changes of potential biomarkers in model rats, and the content of these biomarkers tends to approach the control group (Figure 6). The spatial distribution of PCA scores revealed that rats in the model group could be obviously distinguished from those in the control group, indicating that endogenous regulation alone cannot normalize the metabolic changes in asthma rats to match metabolite levels in control rats after treatment. After the oral administration, the position of these treated rats was far from that of model rats and close to that of the control rats, which indicates that PF was able to normalize the asthma metabolic profile (Figure 7). PF can reverse the abnormal levels of these biomarkers, and the relative levels of biomarkers before and after treatment are shown in Figure 8.

\section{DISCUSSION}

Paeoniflorin is a pinane monoterpene glycoside with various bioactivities, such as anti-oxidative stress, anticancer effects, antiplatelet aggregation, and anti-inflammatory and reducing 


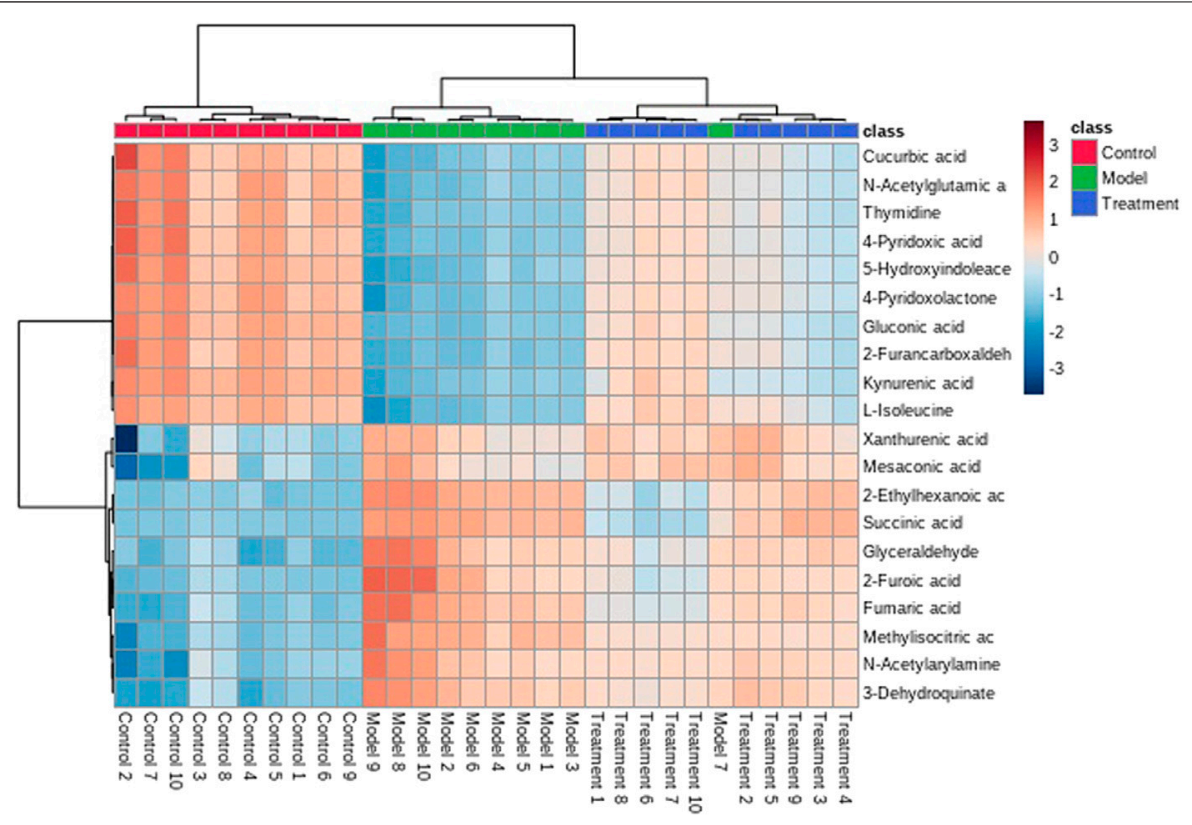

FIGURE 8 | Cluster analysis of the differential metabolites in the control, model, and treatment groups.

blood viscosity activity, and these pharmacological effects lay the foundation for PF of being a potential therapeutic agent for several diseases. Interestingly, the previous studies suggested that it possesses promising anticancer activities. Paeoniflorin has gained a large amount of attention for its effect on asthma disease as the growth rate of asthma has increased in recent years. This work will explore the related molecular mechanisms, which would be beneficial for the further exploration and development of this natural compound. However, the current investigations on action mechanisms of PF are lacking of in vivo experiments.

The high-throughput metabolomics could monitor the metabolites' changes in vivo (Liang et al., 2016c; Liang et al., 2016d; Yu et al., 2017; Zhang et al., 2017; Kachroo et al., 2021). The current metabolomic analysis strategy is widely employed in the metabolic mechanism research and discovery of biomarkers (Zhang et al., 2015; Zhang et al., 2016; Liang et al., 2017).

In this study, UPLC-Q/TOF-MS was used to develop an untargeted metabolomics analysis of asthma rats to explore the overall metabolic changes and characterize biomarkers. As a result, a total of 20 biomarkers with VIP $>1$ and normalized abundance significant $p<0.05$ were discovered. It is worth noting that $\mathrm{PF}$ reversed the levels of biomarker metabolites after treatment, which participated in 5 metabolic pathways including the citrate cycle (TCA cycle), pyrimidine metabolism, pentose phosphate pathway, tyrosine metabolism, and tryptophan metabolism. The biomarkers involved in these metabolic pathways play an important role in asthma. There are defects in this experiment. First of all, the current detection technology cannot analyze all the metabolites of the body at the same time and cannot reasonably interpret all the information obtained. It is necessary to further improve the metabolomic analysis detection technology and data processing technology. Further research is needed in terms of their use in clinical diagnosis.

\section{CONCLUSION}

In this study, an advanced, high-sensitivity and high-throughput UPLC-MS was used to prove the PF-possessed therapeutic effects on asthma in some degree. This study explored the mechanism of PF in improving the rat model of asthma by metabolomics; a total of 20 potential biomarkers were identified in asthma rats. In addition, these biochemical indicators were regulated significantly after the treatment of $\mathrm{PF}$, and five related pathways were significantly affected by PF. Our research had demonstrated that PF was effective against asthma. It has provided the scientific evidence for PF treatment for asthma disease.

\section{DATA AVAILABILITY STATEMENT}

The original contributions presented in the study are included in the article/Supplementary Material; further inquiries can be directed to the corresponding authors.

\section{ETHICS STATEMENT}

The animal study was reviewed and approved by the ethics committee of the Fudan University Pudong Medical Center.

\section{AUTHOR CONTRIBUTIONS}

DW, LZ, ZH, YH, YL, LW, and JZ performed the experiments. DW, LZ, ZH, and $\mathrm{YH}$ analyzed the 
metabolome profiling data. YH, YL, and LW provided reagents and revised the manuscript. SC and LL designed the project. DW, LZ, and $\mathrm{ZH}$ wrote the manuscript. All authors reviewed the manuscript.

\section{FUNDING}

This work was supported by grants from the Discipline Construction Plan of Pudong New Area Health and Family Planning Commission (PWZbr 2017-24).

\section{REFERENCES}

Bush, A., Fitzpatrick, A. M., Saglani, S., Anderson, W. C., 3rd, and Szefler, S. J. (2021). Difficult-to-treat Asthma Management in School-Age Children. J. Allergy Clin. Immunol. Pract. S2213-2198 (21), 01288-1295. doi:10.1016/j. jaip.2021.11.010

Chen, L., Zhao, X., Wei, S., Ma, X., Liu, H., Li, J., et al. (2021). Mechanism of Paeoniflorin on ANIT-Induced Cholestatic Liver Injury Using Integrated Metabolomics and Network Pharmacology. Front. Pharmacol. 12, 737630. doi:10.3389/fphar.2021.737630

Chiu, C. Y., Cheng, M. L., Chiang, M. H., Wang, C. J., Tsai, M. H., and Lin, G. (2021). Integrated Metabolic and Microbial Analysis Reveals Host-Microbial Interactions in IgE-Mediated Childhood Asthma. Sci. Rep. 11 (1), 23407. doi:10. 1038/s41598-021-02925-5

Fan, Q., Guan, X., Hou, Y., Liu, Y., Wei, W., Cai, X., et al. (2020). Paeoniflorin Modulates Gut Microbial Production of Indole-3-Lactate and Epithelial Autophagy to Alleviate Colitis in Mice. Phytomedicine 79, 153345. doi:10. 1016/j.phymed.2020.153345

Fang, H., Zhang, A., Zhou, X., Yu, J., Song, Q., and Wang, X. (2020). Highthroughput Metabolomics Reveals the Perturbed Metabolic Pathways and Biomarkers of Yang Huang Syndrome as Potential Targets for Evaluating the Therapeutic Effects and Mechanism of Geniposide. Front. Med. 14 (5), 651-663. doi:10.1007/s11684-019-0709-5

Guo, K., Zhang, Y., Li, L., Zhang, J., Rong, H., Liu, D., et al. (2021). Neuroprotective Effect of Paeoniflorin in the Mouse Model of Parkinson's Disease through asynuclein/protein Kinase C $\delta$ Subtype Signaling Pathway. Neuroreport 32 (17), 1379-1387. doi:10.1097/WNR.0000000000001739

Han, R. T., Kim, S., Choi, K., Jwa, H., Lee, J., Kim, H. Y., et al. (2017). Asthma-like Airway Inflammation and Responses in a Rat Model of Atopic Dermatitis Induced by Neonatal Capsaicin Treatment. J. Asthma Allergy 10, 181-189. doi:10.2147/JAA.S124902

Han, X., Hu, S., Yang, Q., Sang, X., Tang, D., and Cao, G. (2021). Paeoniflorin Ameliorates Airway Inflammation and Immune Response in Ovalbumin Induced Asthmatic Mice: From Oxidative Stress to Autophagy. Phytomedicine 96, 153835. doi:10.1016/j.phymed.2021.153835

Jiao, F., Varghese, K., Wang, S., Liu, Y., Yu, H., Booz, G. W., et al. (2021). Recent Insights into the Protective Mechanisms of Paeoniflorin in Neurological, Cardiovascular, and Renal Diseases. J. Cardiovasc. Pharmacol. 77 (6), 728-734. doi:10.1097/FJC.0000000000001021

Jin, H., Li, J., Zhang, M., Luo, R., Lu, P., Zhang, W., et al. (2021). Berberine-Loaded Biomimetic Nanoparticles Attenuate Inflammation of Experimental Allergic Asthma via Enhancing IL-12 Expression. Front. Pharmacol. 12, 724525. doi:10. 3389/fphar.2021.724525

Kachroo, P., Sordillo, J. E., Lutz, S. M., Weiss, S. T., Kelly, R. S., McGeachie, M. J., et al. (2021). Pharmaco-Metabolomics of Inhaled Corticosteroid Response in Individuals with Asthma. J. Pers Med. 11 (11), 1148. doi:10.3390/jpm11111148

Koh, L., Grigg, J., and Whitehouse, A. (2021). Personal Monitoring to Reduce Exposure to Black Carbon in Children with Asthma: a Pilot Study. ERJ Open Res. 7 (4), 00482-02021. doi:10.1183/23120541.00482-2021

Liang, Q., Wang, C., Li, B., and Zhang, A.-h. (2015a). Metabolomics of Alcoholic Liver Disease: a Clinical Discovery Study. RSC Adv. 5 (98), 80381-80387. doi:10.1039/c5ra13417j

\section{ACKNOWLEDGMENTS}

The authors thank all members of the laboratory for insightful discussions.

\section{SUPPLEMENTARY MATERIAL}

The Supplementary Material for this article can be found online at: https://www.frontiersin.org/articles/10.3389/fphar.2022.829780/ full\#supplementary-material

Liang, Q., Liu, H., Zhang, T., Jiang, Y., Xing, H., and Zhang, A.-h. (2015b). Metabolomics-based Screening of Salivary Biomarkers for Early Diagnosis of Alzheimer's Disease. RSC Adv. 5 (116), 96074-96079. doi:10.1039/c5ra19094k

Liang, Q., Liu, H., Zhang, T., Jiang, Y., Xing, H., and Zhang, A.-h. (2016a). Discovery of Serum Metabolites for Diagnosis of Progression of Mild Cognitive Impairment to Alzheimer's Disease Using an Optimized Metabolomics Method. RSC Adv. 6 (5), 3586-3591. doi:10.1039/c5ra19349d

Liang, Q., Liu, H., Li, X., and Zhang, A.-H. (2016b). High-throughput Metabolomics Analysis Discovers Salivary Biomarkers for Predicting Mild Cognitive Impairment and Alzheimer's Disease. RSC Adv. 6 (79), 75499-75504. doi:10.1039/c6ra16802g

Liang, Q., Liu, H., Xing, H., Jiang, Y., and Zhang, A.-H. (2016c). Urinary UPLC-MS Metabolomics Dissecting the Underlying Mechanisms of Huaxian Capsule Protects against Sepsis. RSC Adv. 6 (46), 40436-40441. doi:10.1039/c6ra07987c

Liang, Q., Liu, H., Xing, H., Jiang, Y., and Zhang, A.-H. (2016d). UPLC-QTOF/MS Based Metabolomics Reveals Metabolic Alterations Associated with Severe Sepsis. RSC Adv. 6 (49), 43293-43298. doi:10.1039/c6ra07514b

Liang, Q., Liu, H., Xie, L.-X., Li, X., and Zhang, A.-H. (2017). High-throughput Metabolomics Enables Biomarker Discovery in Prostate Cancer. RSC Adv. 7 (5), 2587-2593. doi:10.1039/c6ra25007f

Liu, R., Zheng, Y., Han, T., Lan, J., He, L., and Shi, J. (2021). Angiogenic Actions of Paeoniflorin on Endothelial Progenitor Cells and in Ischemic Stroke Rat Model. Am. J. Chin. Med. 49 (4), 863-881. doi:10.1142/S0192415X21500415

Ma, J., Meng, Q., Zhan, J., Wang, H., Fan, W., Wang, Y., et al. (2021). Paeoniflorin Suppresses Rheumatoid Arthritis Development via Modulating the CircFAM120A/miR-671-5p/MDM4 Axis. Inflammation 44 (6), 2309-2322. doi:10.1007/s10753-021-01504-0

Qiu, S., Zhang, A.-h., Guan, Y., Sun, H., Zhang, T.-l., Han, Y., et al. (2020). Functional Metabolomics Using UPLC-Q/TOF-MS Combined with Ingenuity Pathway Analysis as a Promising Strategy for Evaluating the Efficacy and Discovering Amino Acid Metabolism as a Potential Therapeutic MechanismRelated Target for Geniposide against Alcoholic Liver Disease. RSC Adv. 10 (5), 2677-2690. doi:10.1039/c9ra09305b

Shou, Q., Jin, L., Lang, J., Shan, Q., Ni, Z., Cheng, C., et al. (2019). Integration of Metabolomics and Transcriptomics Reveals the Therapeutic Mechanism Underlying Paeoniflorin for the Treatment of Allergic Asthma. Front. Pharmacol. 9, 1531. doi:10.3389/fphar.2018.01531

Sun, H., Zhang, A. H., Yang, L., Li, M. X., Fang, H., Xie, J., et al. (2019). Highthroughput Chinmedomics Strategy for Discovering the Quality-Markers and Potential Targets for Yinchenhao Decoction. Phytomedicine 54, 328-338. doi:10.1016/j.phymed.2018.04.015

Wei, S., Ma, X., Niu, M., Wang, R., Yang, T., Wang, D., et al. (2020). Mechanism of Paeoniflorin in the Treatment of Bile Duct Ligation-Induced Cholestatic Liver Injury Using Integrated Metabolomics and Network Pharmacology. Front. Pharmacol. 11, 586806. doi:10.3389/fphar.2020.586806

Werder, R. B., Ullah, M. A., Rahman, M. M., Simpson, J., Lynch, J. P., Collinson, N., et al. (2021). Targeting the P2Y13 Receptor Suppresses IL-33 and HMGB1 Release and Ameliorates Experimental Asthma. Am. J. Respir. Crit. Care Med. Online ahead of print. doi:10.1164/rccm.202009-3686OC

Wu, X., Qi, X., Wang, J., Zhang, Y., Xiao, Y., Tu, C., et al. (2021). Paeoniflorin Attenuates the Allergic Contact Dermatitis Response via Inhibiting the IFN- $\gamma$ Production and the NF-Kb/ikba Signaling Pathway in T Lymphocytes. Int. Immunopharmacol 96, 107687. doi:10.1016/j.intimp.2021.107687 
Xie, J., Zhang, A. H., Qiu, S., Zhang, T. L., Li, X. N., Yan, G. L., et al. (2019). Identification of the Perturbed Metabolic Pathways Associating with Prostate Cancer Cells and Anticancer Affects of Obacunone. J. Proteomics 206, 103447. doi:10.1016/j.jprot.2019.103447

Yu, J., Kong, L., Zhang, A., Han, Y., Liu, Z., Sun, H., et al. (2017). HighThroughput Metabolomics for Discovering Potential Metabolite Biomarkers and Metabolic Mechanism from the APPswe/PS1dE9 Transgenic Model of Alzheimer's Disease. J. Proteome Res. 16 (9), 3219-3228. doi:10.1021/acs.jproteome.7b00206

Yu, W., Zeng, M., Xu, P., Liu, J., and Wang, H. (2021). Effect of Paeoniflorin on Acute Lung Injury Induced by Influenza A Virus in Mice. Evidences of its Mechanism of Action. Phytomedicine 92, 153724. doi:10.1016/j.phymed.2021.153724

Zhang, A., Wang, H., Sun, H., Zhang, Y., An, N., Yan, G., et al. (2015). Metabolomics Strategy Reveals Therapeutical Assessment of Limonin on Nonbacterial Prostatitis. Food Funct. 6 (11), 3540-3549. doi:10.1039/ c5fo00489f

Zhang, A., Sun, H., Yan, G., Wang, P., and Wang, X. (2016). Mass Spectrometry-Based Metabolomics: Applications to Biomarker and Metabolic Pathway Research. Biomed. Chromatogr. 30 (1), 7-12. doi: $10.1002 /$ bmc. 3453

Zhang, A., Sun, H., and Wang, X. (2017). Emerging Role and Recent Applications of Metabolomics Biomarkers in Obesity Disease Research. RSC Adv. 7, 14966-14973. doi:10.1039/c6ra28715h

Zhang, A. H., Ma, Z. M., Sun, H., Zhang, Y., Liu, J. H., Wu, F. F., et al. (2019a). High-Throughput Metabolomics Evaluate the Efficacy of Total Lignans from Acanthophanax Senticosus Stem against Ovariectomized Osteoporosis Rat. Front. Pharmacol. 10, 553. doi:10.3389/fphar.2019.00553

Zhang, A.-H., Sun, H., Yan, G.-L., Han, Y., Zhao, Q.-Q., and Wang, X.-J. (2019b). Chinmedomics: A Powerful Approach Integrating Metabolomics with Serum
Pharmacochemistry to Evaluate the Efficacy of Traditional Chinese Medicine. Engineering 5, 60-68. doi:10.1016/j.eng.2018.11.008

Zhang, A. H., Ma, Z. M., Kong, L., Gao, H. L., Sun, H., Wang, X. Q., et al. (2020). High-throughput Lipidomics Analysis to Discover Lipid Biomarkers and Profiles as Potential Targets for Evaluating Efficacy of Kai-Xin-San against APP/PS1 Transgenic Mice Based on UPLC-Q/TOF-MS. Biomed. Chromatogr. 34 (2), e4724. doi:10.1002/bmc.4724

Zhao, Y., Li, X., Chu, J., Shao, Y., Sun, Y., Zhang, Y., et al. (2021). Inhibitory Effect of Paeoniflorin on IgE-dependent and IgE-independent Mast Cell Degranulation In Vitro and Vivo. Food Funct. 12 (16), 7448-7468. doi:10. 1039/d1fo01421h

Conflict of Interest: The authors declare that the research was conducted in the absence of any commercial or financial relationships that could be construed as a potential conflict of interest.

Publisher's Note: All claims expressed in this article are solely those of the authors and do not necessarily represent those of their affiliated organizations, or those of the publisher, the editors, and the reviewers. Any product that may be evaluated in this article, or claim that may be made by its manufacturer, is not guaranteed or endorsed by the publisher.

Copyright (c) 2022 Wang, Zhao, Hao, Huang, Liao, Wang, Zhang, Cao and Liu. This is an open-access article distributed under the terms of the Creative Commons Attribution License (CC BY). The use, distribution or reproduction in other forums is permitted, provided the original author(s) and the copyright owner(s) are credited and that the original publication in this journal is cited, in accordance with accepted academic practice. No use, distribution or reproduction is permitted which does not comply with these terms. 